\title{
Ecare@Home: A Distributed Research Environment on Semantic Interoperability
}

Amy Loutfi, Arne Jönsson, Lars Karlsson, Leili Lind, Maria Linden, Federico Pecora and Thiemo Voigt

The self-archived postprint version of this journal article is available at Linköping University Institutional Repository (DiVA):

http:// urn.kb.se/ resolve?urn=urn:nbn:se:liu:diva-141052

N.B.: When citing this work, cite the original publication.

The original publication is available at www.springerlink.com:

Loutfi, A., Jönsson, A., Karlsson, L., Lind, L., Linden, M., Pecora, F., Voigt, T., (2016), Ecare@Home: A Distributed Research Environment on Semantic Interoperability, Internet of Things Technologies for HealthCare, , 3-8. https:/ / doi.org/ 10.1007/9783-319-51234-1_ 1

Original publication available at:

https:// doi.org/ 10.1007/978-3-319-51234-1_1

Copyright: Springer Verlag

http:// www.springerlink.com/?MUD=MP 


\title{
Ecare@Home: A Distributed Research Environment on Semantic Interoperability
}

\author{
Amy Loutfi ${ }^{1}$, Arne Jönsson ${ }^{2}$, Lars Karlsson ${ }^{1}$, Leili Lind ${ }^{2}$ Maria Linden ${ }^{3}$, \\ Federico Pecora ${ }^{1}$, and Thiemo Voigt ${ }^{4}$ \\ 1 Örebro University, Örebro, Sweden \\ firstname. lastname@oru.se, \\ 2 SICS East, Linköping, Sweden \\ firstname. lastname@sics.se \\ 3 Mälardalen University, Västerås, Sweden \\ firstname. lastname@mdh.se \\ 4 SICS ICT, Stockholm, Sweden \\ firstname. lastname@sics.se
}

\begin{abstract}
This paper presents the motivation and challenges to developing semantic interoperability for an internet of things network that is used in the context of home based care. The paper describes a research environment which examines these challenges and illustrates the motivation through a scenario whereby a network of devices in the home is used to provide high-level information about elderly patients by leveraging from techniques in context awareness, automated reasoning, and configuration planning.
\end{abstract}

Key words: semantic interoperability, configuration planning, health and care, internet of things

\section{Introduction}

A current vision in the area of ICT-supported independent living of the elderly involves populating the home with connected electronic devices ("things") such as sensors and actuators and linking them to the Internet. Creating such an Internet-of-Things infrastructure (IoT) is done with the ambition to provide automated information gathering and processing on top of which e-services can be built for the elderly residing in their homes [2]. Technically speaking IoT is mainly supported by continuous progress in wireless sensor/actuator networks software applications and by manufacturing low cost and energy efficient hardware for device communications. However, there is still the major the major challenge for expanding generic IoT technologies to efficient ICT-supported services for the elderly. Namely, services such as the personal health record (PHR) and other computerized "things" such as care and health related information resources, i.e., electronic health records (EHR), home-service (in Swedish: hemtjänst) documentation, end-user generated information, informal care-givers related information (e.g., information provided from family, neighbors, social networks etc.) must be integrated with the IoT infrastructure. 
Ecare@home is a distributed research environment whose aim is to address the above vision by 1 ) performing research on selected fundamental issues in semantic interoperability with a particular focus on human-machine interoperability, that is to say how to enable users to query and control the IoT infrastructure on meaningful terms that are human interpretable and EHR/PHR compatible.; and 2) testing the research results on a technical platform which is embedded in the Internet of Things and provides information with an unambiguous, shared meaning across IoT devices, elderly residents, relatives, health-and-care professionals and organizations and various personal information repositories and the various electronic health records associated with those.

This paper describes the main motivations and challenges which the research environment will address. It provides a brief overview of the various types of interoperability and then follows the main challenges that will be addressed within the scope of the research environment. A short scenario and illustrative scenario is outlined to conclude the paper.

\section{The Dimensions of Interoperability}

Generally speaking, interoperability is "the ability of two or more systems or components to exchange data and use information". Interoperability is a major concern of many organizations, including the European Commission which lists interoperability as a pillar in the Digital Agenda for Europe. This definition provides many challenges on how to 1) Get the data/information 2) Exchange data/information, and 3) Use the data/information in understanding it and being able to process it. There are different types of interoperability: technical, syntactical, semantic, and organizational. E-care@home focusses on semantic interoperability, still the planned research is expected to also impact the other types.

Semantic interoperability is seen as a key requirement for gaining the benefits of computerization of the health domain and much effort has been invested by both national programs and academia to understand and provide solutions for the problem of achieving semantic interoperability. Semantic interoperability is usually associated with the meaning of content, information or data, and concerns both the human and machine interpretation of content [4]. The focus here is on the semantic annotation of the data (for example, with domain knowledge) which can provide machine-interpretable descriptions on what the data represents, and meta-data such as where it originates from, how it can be related to its surroundings, what is providing it, and what are the quality, technical, and non-technical attributes. This semantic interpretation can be used for machine to machine communication (M2M). An example of M2M semanticsaugmented communication would be a sensor measuring room temperature on demand of an end-user and, in relation to other information possibly available about the end-user, setting the temperature to an adequate level. Devices and sensors register through network gateway which writes their data into semantic database. Every time the sensor sends the temperature, the gateway writes it 
into the repository, and matches it with desired temperature inside the room where sensor is located. For example, the database could also keep information about the preferences of the user and contextual information needed to make room temperature decisions. However, another central aspect of semantic interoperability concerns the human interpretation of data and information provided by the IoT devices or by other humans respectively. Thus, interoperability on this level means that there is a common understanding between people and between people and devices of the meaning of the content being communicated. Regarding this type of interoperability E-care@home focuses on the contextualised semantic annotation of uncertain sensor / actuator data using symbolic reasoning methods. E-care@home also focuses on formal methods for modelling contextualised information from heterogeneous data sources so that a common understanding is achieved with the help of various mechanisms studied in the project, for instance ontology alignment. Ontologies not only provide some level of semantic expressiveness to the information they also allow the information exchange between applications and between different levels of abstraction.

The E-care@home research environment is needed to address an evitable and emerging challenge in sensor deployment in home and health environments. In short, this challenge entails adding value of raw sensor data by understanding the meaning of this data and involves collection, modelling, reasoning and distribution of context in relation to sensor data.

\section{Semantic Modelling and Ontologies}

Ontologies are tools for specifying the semantics of terminology systems in a well defined and unambiguous manner. Ontologies are used to improve communication either between humans or computers by specifying the semantics of the symbolic apparatus used in the communication process. The domain of application, here the health domain, is often significantly more complex than what practically usable ontologies might express. E.g. common health domain use cases require second-order reasoning which is not available in, e.g., any of the OWL profilesvii. Typically, use cases' requirements have to be balanced with computational effectiveness requirements and approximate solutions have to be developed and maintained. Thus, large-scale biomedical ontologies, such as SNOMED CT and NCI Thesaurus, are often using low-expressivity logics to allow to perform little reasoning, while smaller ontologies might allow higher expressivity. For enabling interoperability, all applications and users must share a common terminology. If an application uses a terminology different from another then a mapping between different ontologies must be made. A challenge is that in an care setting with an Iot infrastructure, the types of ontologies currently available are still disparate: on one hand, there is a growing establishment of ontologies for describing sensors and their observations such as the SSN; on the other hand, there are the ontologies in the health domain that capture relations between medical terms providing codes, terms, synonyms and definitions used in clinical documentation and reporting. Thus, there is a need to study these 
ontologies in a holistic context, e.g., a network of ontologies by reusing existing ones and alignments between those.

\section{Context Reasoning in an Ecare@home setting}

Ontologies and other semantic technologies can be key enabling technologies for sensor networks because they will improve semantic interoperability and integration, as well as facilitate reasoning and classification. Still, significant effort has yet to be placed on how to integrate the information in ontologies or other semantic models with formal reasoning methods specifically for improved sensor interpretation/annotation. Some work to this effect has been discussed in [Barghani et al]. The domains considered so far deal with e.g. weather data where rule-based reasoning is relatively straight forward. Integrating proper reasoning techniques in more complex domains such as the one envisioned in Ecare@home is a more difficult problem. Sensors can only measure limited physical phenomenon and sensor data is inherently uncertain. Therefore, eventual reasoning methods must be also contending with the uncertain nature of the sensor data [3]. Context and patient profiling also plays a crucial role, and therefore the reasoner should make use of contextual information in a meaningful way. Finally, trust in the system is crucial for uptake, and enabling trust is often done via transparency, thus requiring explication and readability of the reasoner's output. Given these requirements, E-care@home will explore reasoning techniques e.g. answer set programming, for semantic-web data, focusing in particular on abductive reasoning methods that are non-monotonic in nature [5]. In part this has been examined in limited contexts of smart home networks, but including diverse semantic models as mentioned above is still a remaining challenge.

\section{Semantic Interoperability for Service Discovery}

Semantic interoperability not only enables a Machine-to-Human interaction but also enables a Human-to-Machine interaction, where in this case, human requests for services can transcend to machine readable code. High-level tasks requested by users such as "measure physical activity" should lead to a number of services being activated which also may relate to a number of devices which should provide the necessary data. In E-care@home we choose to see service composition as a configuration planning problem where configuration planning generates a functional configuration of a networked system consisting both of sensors and actuators distributed in the environment that solves a given task. In a functional configuration, sensory, computational and motoric functionalities belonging to the different devices are connected with communication channels. Another related and complementary area is web service composition. Web service composition, while not directly involving physical sensors and actuators, is concerned with ways of interconnecting selfcontained, self-describing, modular applications that can be published, located, and invoked across the web. 
A composite service is a set of services and the control and data flow among them. A number of approaches have been explored, from genetic algorithms, to neo-classical planning, and semantic information is often used for representing the domain. The challenge investigated in an E-care@home domain is to develop methods for service composition that not only fulfill hard constraints but also take into account preferences. Solving this challenge means that requests and queries can reconfigure device networks, thus adding robustness and reliability to obtaining information [7].

\section{Testbeds and Scenario}

An important objective of E-care@home is to evaluate the developed methods in real contexts. This includes a number of living lab environments but also as the environments progress we will include real test bed environments (e.g., IoT enhanced homes) where such test beds enable reliable data collection. For this objective to be met, synergies between existing projects and test beds will be made. Also, in order to properly verify results, test homes will be selected by first forming adequate use cases which outline the requirements for each test site and test persons involved in the evaluation. Figure 6 revisits the scenario outlined with respect to the above scientific objectives. In this Figure, a healthcare professional may want to query for specific information. This query probes one or several service(s) where the service requires that a particular configuration of devices in the home is active. If certain sensors were not activated (not shown in this example) they could be actuated by the configuration planner. Also the configuration planner takes into account preferences and not only hard constraints. To derive answers to queries or to infer information, the reasoning module uses information both from the sensors (and eventual signal processing) as well from the models to contextualize the interpretation. Multiple explanations are generated and in particular, sensor uncertainties are factored into the final output. This example is illustrative but emphasizes an important aspect that semantic AI techniques can be used to automate processes that configure, infer, and manage knowledge emerging from the sensor/actuator devices and, provide a meaningful output of the entire system.

\section{Conclusion}

This discussion paper presented a research environment Ecare@home whose objective is to rely on an IoT framework in order to gather information about inhabitants, abstract that information, and communicate it in a human consumable way to the various users of the system. Thus in E-care@home we aim to achieve something very different from the previous works in context-aware IoT and we aim to shift focus from network centric context awareness to data centric context awareness. The IoT notion is still very important as it is the setting for which the E-care@home domain exists. However, what is important 


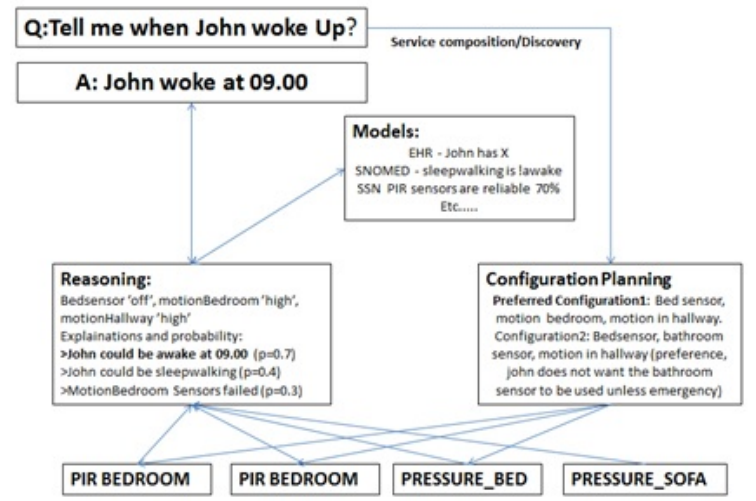

Fig. 1. An illustrative scenario which highlights the various challenges and motivations in achieving a semantic intereroperability between an IoT network and the various types of users in a home-based care setting.

in E-care@home is the data, observations and measurements and not the node that provides it. E-care@home embedded in an IoT will go beyond state of the art by bringing data-centric approach to IoT. Focus is put on interpretation of data, which is, translating sensor data to knowledge that is usable by people, organisations and applications. This involves abstraction whereby low-level data is converted to high-level knowledge and where prior knowledge and reasoning can interpret the data and infer beyond that which is physically measured.

\section{References}

1. C. Perera, A. Zaslavsky, P. Christen, D. Georgakopoulos Context Aware Computing for The Internet of Things: A Survey. IEEE Communications Surveys \& Tutorials Journal, 2013

2. Riboni, D., Bettini, C.: Context-aware activity recognition through a combination of ontological and statistical reasoning. In: Proceedings of the 6th International Conference on Ubiquitous Intelligence and Computing. pp.39-53. UIC '09, SpringerVerlag, Berlin, Heidelberg (2009)

3. D. Kalra. Barriers, approaches and research priorities for semantic interoperability in support of clinical care delivery. SemanticHEALTH deliverable 4.1.

4. M. Alirezaie, A. Loutfi. Reasoning for Improved Sensor Data Interpretation in a Smart HomeARCOE-Logic 2014, International Workshop on Acquisition, Representation and Reasoning about Context with Logic. November 24-25, 2014. Linkoping, Sweden.

5. M. Baldauf, S. Dustdar, and F. Rosenberg, A survey on context aware systems, Int. J. Ad Hoc Ubiquitous Comput., vol. 2, no. 4,pp. 263-277, Jun. 2007.

6. L. Silva-Lopez, M Broxvall, A Loutfi, L Karlsson. Towards Configuration Planning with Partially Ordered Preferences: Representation and Results. KI-Künstliche Intelligenz 29 (2), 173-183 